\title{
artigo
}

Rocha, M.A.; Temoteo, R.C.A.; Figueiredo, C.R.F.; Pereira da Silva, R.R.; Menezes, T.I.M.; Costa, P.M.B.;

Importância do tratamento diretamente observado da tuberculose na percepção dos enfermeiros

\section{Importância do tratamento diretamente observado da tuberculose na percepção dos enfermeiros}

\author{
Importance of treatment directly observed of tuberculosis in the perception of nurses \\ Importancia del tratamiento directamente observado de la tuberculosis en la percepción de las enfermeras
}

\begin{abstract}
RESUMO
Objetivo: Este estudo objetivou avaliar a importância da realização do Tratamento Diretamente Observado da tuberculose (TDO) na percepção dos enfermeiros das unidades de saúde da família no município de Cajazeiras-PB. Métodos: Trata-se de um estudo de campo do tipo exploratório descritivo com abordagem qualitativa. A pesquisa ocorreu nas Unidades de Saúde da Família do município de Cajazeiras. Como técnica para coleta de dados, foi utilizada uma entrevista semiestruturada. Resultados: Foram divididos em duas categorias: dados sócio demográficos e importância na realização do TDO. Participaram da pesquisa 18 enfermeiros, destes 2 eram homens e 16 mulheres. A maioria dos profissionais tinham mais de 5 anos de atividade profissional e possuíam pós graduação na área de atuação. Além disso, a maioria enfatizou que é importante o TDO pela formação do vínculo com usuário e a consequente prevenção do abandono ao tratamento. Conclusão: A realização deste estudo proporcionou um maior conhecimento a respeito da importância pela perspectiva dos entrevistados, pois através do vínculo da equipe com o doente de tuberculose é possivel realizar o monitoramento das tomadas, identificar os fatores adversos, evitar o abandono e principalmente acompanhar a evolução.
\end{abstract}

DESCRITORES: Tuberculose; Vigilância; Atenção primária à saúde; Enfermeiros de Saúde da Família.

\section{ABSTRACT}

Objective: This study aimed to evaluate the importance of performing the Directly Observed Treatment of Tuberculosis (DOT) in the perception of nurses in family health units in the city of Cajazeiras-PB. Methods: This is a descriptive exploratory field study with a qualitative approach. The research took place in Family Health Units in the municipality of Cajazeiras. As a technique for data collection, a semi-structured interview was used. Results: They were divided into two categories: socio-demographic data and importance in carrying out the DOT. Eighteen nurses participated in the research, of which 2 were men and 16 women. Most professionals had more than 5 years of professional activity and had postgraduate degrees in the area of expertise. In addition, the majority emphasized that DOT is important due to the formation of a bond with the user and the consequent prevention of abandonment of treatment. Conclusion: This study provided more knowledge about the importance from the perspective of the interviewees, because through the link between the team and the tuberculosis patient it is possible to monitor the take, identify the adverse factors, avoid abandonment, and especially follow the evolution.

DESCRIPTORS: Tuberculosis; Surveillance; Primary health care; Family Nurse Practitioners.

\section{RESUMEN}

Objetivos: Este estudio tenía como objetivo evaluar la importancia de realizar el tratamiento directamente observado de la tuberculosis (TDO) en la percepción de enfermeras en unidades de salud familiar en la ciudad de Cajazeiras-PB. Métodos: Se trata de un estudio de campo exploratorio descriptivo con enfoque cualitativo. La investigación se llevó a cabo en Unidades de Salud de la Familia del municipio de Cajazeiras. Como técnica para la recolección de datos se utilizó una entrevista semiestructurada. Resultados: Se dividieron en dos categorías: datos sociodemográficos e importancia en la realización del TDO. En la investigación participaron 18 enfermeros, de los cuales 2 eran hombres y 16 mujeres. La mayoría de los profesionales tenían más de 5 años de actividad profesional y tenían títulos de posgrado en el área de especialización. Además, la mayoría enfatizó que el TDO es importante debido a la formación de un vínculo con el usuario y la consecuente prevención del abandono del tratamiento. Conclusión: Este estudio proporcionó un mayor conocimiento sobre la importancia desde la perspectiva de los entrevistados, ya que a través de la relación del equipo con el paciente tuberculoso, es posible monitorear la toma, identificar los factores adversos, evitar el abandono y, especialmente, seguir la evolución.

DESCRIPTORES: Tuberculosis; Vigilancia; Atención primaria de salud; Enfermeras de Familia.

RECEBIDO EM: 30/01/2021 APROVADO EM: 16/02/2021 


\section{Mariana de Araujo Rocha}

Graduação em Enfermagem pela Universidade Federal de Campina Grande e Pós- Graduada em Saúde Pública com Ênfase em Saúde da Família - Uninter.

ORCID: 0000-0002-0650-8062

\section{Rayrla Cristina de Abreu Temoteo}

Enfermeira pela Universidade Federal de Campina Grande, Especialista em Saúde da Família e Saúde Coletiva, Mestre em Saúde Pública pela PPGS/UEPB, Doutoranda em Enfermagem pela PGENF/UFRN.

ORCID: 0000-0003-1980-7819

\section{Camila Rolim Figueiredo}

Enfermeira pela Universidade Federal de Campina Grande, Pós-graduanda em Enfermagem em Centro Cirúrgico pela Universidade de Pernambuco.

ORCID: 0000-0003-4704-8653

\section{Rayanne Rilka Pereira da Silva}

Enfermeira pela Universidade Federal de Campina Grande, Pós-graduada em Saúde da Família pela Universidade Candido Mendes.

ORCID: 0000-0003-1851-6446

\section{Thaline Ingrid Marques Menezes}

Enfermeira pela Universidade Federal de Campina Grande, Pós-graduada em UTI, Urgencia e Emergencia. ORCID: 0000-0001-5123-3232

\section{Paula Micaelle Barbosa Costa}

Enfermeira pela Universade Federal de Campina Grande e pós-graduada em Obstetrícia e Neonatologia.

ORCID: 0000-0002-0765-5873

\section{INTRODUÇÃO}

A tuberculose (TB), embora com reconhecido agente causal e tratamentos estabelecidos de longa data, persiste como relevante problema de Saúde Pública, sobretudo em países em desenvolvimento. ${ }^{1}$ Trata-se de uma doença infecciosa, causada pela bactéria Mycobacterium Tuberculosis. ${ }^{2}$

O Brasil, encontra-se entre os países com cargas mais elevadas da doença, com taxa de incidência de 34,8 casos por 100 mil habitantes em 2018, uma vez que tem se distanciado da taxa preconizada pela Organização Mundial da Saúde (OMS), de 10 casos/100 mil hab., o que ressalta a necessidade de avanços para o alcance da meta da OMS. ${ }^{3}$

Em todo o mundo, os Programas de Controle da Tuberculose, orientados pela estratégia Stop TB - implementada no período de 1990 a 2015 para eliminar a tuberculose como um problema de saúde pública - recomendam a implantação e sustentabilidade da estratégia Directly Ob- served Treatment Short-Course (DOTS) objetivando melhorar as condições de diagnóstico laboratorial, tratamento sob supervisão com fornecimento contínuo dos fármacos e um sistema de informação e registro adequado para monitorar o desenvolvimento do Programa. ${ }^{4,5}$

O DOTS consiste na observação da ingesta dos medicamentos, estratégia que visa à adesão do indivíduo. Devem ser supervisionadas pelo menos 72 doses de medicação padronizada em duas fases do tratamento: 24 na primeira e 48 doses na segunda. ${ }^{6}$

Esses medicamentos vêm sendo utilizados de modo inadequado ou o paciente abandona por mais de trinta dias, principalmente com a constatação da melhora clínica e constituem duas condições que acentuam as dificuldades na eliminação da doença. Ainda que o Ministério da Saúde (MS) tenha adotado o DOTS, essa estratégia se diferencia por oportunizar uma abordagem singularizada, demonstra considerável progresso, mas os coeficientes de abandono ainda permanecem elevados. ${ }^{4}$
O DOTS assegura um vínculo entre o serviço de saúde, o doente e a família, identificando grupos de risco para a não adesão ao tratamento, propiciando uma aproximação dos profissionais com os indivíduos e seu contexto social. Essa estratégia ajuda a desviar barreiras que dificultam a adesão ao tratamento, devendo desenvolver um plano para as demandas encontradas. ${ }^{7}$

Justifica-se o presente estudo devido a relevância da supervisão do tratamento da TB por papel do profissional de enfermagem, uma vez que estes são os que realizam o diagnóstico e acompanham os paciente durante todo o tratamento até a alta por cura. Insto contribuirá para o controle da doença, aumentando as chances de cura e reduzindo o número de casos de abandono, o que servirá de base para pesquisas, a fim de colaborar para a melhoria da assistência prestada.

Sendo assim, os profissionais da atenção básica devem ter uma atitude de vigilância em relação a importância de suas ações na realização do DOTS da TB. Diante do exposto, a questão que norteou a execução 
dessa pesquisa foi: qual a importância da realização do DOTS sob a percepção dos enfermeiros em Unidades de Saúde da Família do município de Cajazeiras-PB?

\section{MÉTODO}

Trata-se de um estudo de campo do tipo exploratório descritivo com abordagem qualitativa, realizado com enfermeiros de Unidades de Saúde da Família (USF) que atenderam a pessoas com tuberculose.

A população do estudo é composta por 23 profissionais enfermeiros de USFs do município de Cajazeiras- PB na zona rural e urbana. A amostra foi composta pelos enfermeiros que concordaram em participar da pesquisa e preencheram os critérios de inclusão do estudo.

Definiu-se como critérios de inclusão adotado para o estudo que o enfermeiro estivesse exercendo as atividades laborais no período da pesquisa e como critérios de exclusão, estar de férias ou afastado e licença-saúde.

A coleta de dados foi realizada nas respectivas USF, após agendamento prévio de datas e horários para execução da mesma de acordo com a disponibilidade dos

Tabela 1. Descrição da categoria 2 correlacionadas as respectivas subcategorias e frequência de acordo com as falas dos entrevistados. Cajazeiras - Paraiba, 2016.

\section{SUBCATEGORIA}

Acompanhamento da tomada da medicação

Identificação de efeitos adversos

Melhoria da assistência prestada ao paciente

Interrupção da cadeia de transmissão
"[...] tratamento é importante ser observado, porque eu vou saber se o paciente está tomando o medicamento[...]" (E - 1)

"[...]Também de ter um controle das tomadas, saber que o paciente está tomando da forma correta [...] (E - 16)

"[...] nos ajuda a identificar se o paciente está com efeitos adversos[...]" (E - 4)

"[...] porque tem muito paciente que apresenta dores gástricas, então tem que orienta-lo quanto a alimentação, e também o acompanhamento né [...]" (E -5)

"Porque eu vou proporcionar uma assistência de melhor qualidade ao paciente, e ficar mais próximo da comunidade, né o PSF e o profissional. (E - 10)

"[...] acho de grande importância porque melhora a atenção ao paciente[...]" (E-6)

"Considero importante porque evita o abandono e a contaminação de outras pessoas, evitando a cadeia de transmissão [...]" (E-4)

"[...] a questão de interromper a transmissão para outras pessoas." (E-6) ficação, categorização e inferência. ${ }^{8}$ Após transcrever as entrevistas, foram visualizadas as falas, em seguida, agrupadas em categorias de análise (método de análise por categorias temáticas).

O projeto dessa pesquisa foi aprovado pelo Comitê de Ética em Pesquisa do Centro de Formação de Professores-Universidade Federal de Campina Grande sob CAAE no 58915116.1.0000.5182 e número do parecer 1.756.039.

\section{RESULTADOS}

A seguir serão apresentados os resultados alcançados através do roteiro de entrevista semiestruturada. Os dados foram divididos em duas categorias, a primeira referente aos dados sócio demográficos e na segunda categoria, foram apresentados os resultados da pesquisa diante das falas dos enfermeiros a respeito da importância da realização do TDO nas USF.

\section{Categoria 1- Caracterização sociode- mográfica}

Participaram da pesquisa 18 dos 23 enfermeiros da ESF do município de Cajazeiras - PB. Dos 18 entrevistados 2 eram homens e 16 mulheres; idades variavam de $25-30$ anos (27,8 \%), 31-40 (61,1\%), 41-60 (11,1\%); destes 38,9\% eram solteiros e $44,4 \%$ casados. Em relação a tempo de atividade profissional menos de 1 ano (5,5\%), $1-5$ anos (38,9\%), 6-10 anos (38,9\%), $11-22$ anos (16,7\%), destes 61,1 $\%$ tinham pós graduação na área de atuação e ainda $61,1 \%$ tinham também outro vínculo empregatício.

\section{Categoria 2- importância da realização do TDO na percepção dos enfermeiros}

Nessa etapa foi avaliado qual a percepção dos entrevistados sobre o TDO, o porque eles consideravam importante realizá-lo e os motivos da importância desse momento, conforme a tabela apresentada a seguir.

Conforme a visão dos enfermeiros entrevistados acima, foi possível evidenciar que os profissionais possuem conhecimento sobre importância do TDO. Visto que a maioria ressaltou que esse momento é 


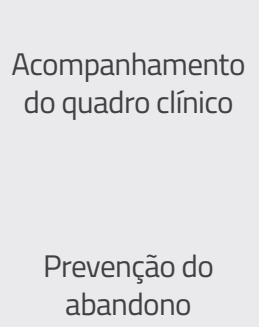

Prevenção da resistência medicamentosa

Total
"[...]E também ajuda aos profissionais acompanharem a evolução da doença, se a medicação está fazendo efeito." (E-4)

"[...] tem como a gente ter esse acompanhamento maior, se é um paciente que está evoluindo bem [...]" (E-12)

"Sim. Eu considero importante porque é um meio da pessoa não... não abandonar o tratamento[...]" (E-2)

"É importante assim, para não desestimular, diante das primeiras melhoras ele não desistir, para ele não abandonar [...]". (E-7)

"[...]não toma direito e acontece essa resistência né, a isoniazida principalmente [...]" (E-9)

"[...]ajuda a ele não abandonar o tratamento, evitando complicações como a resistência medicamentosa né." (E-11) fundamental por favorece o vínculo da equipe com o doente por tuberculose, além da possibilidade de saber se o paciente está fazendo uso da medicação, como também da realização do monitoramento da tomada dessas doses, identificar os fatores adversos e resistência a alguma das drogas. Ressaltou-se também que estes são fatores primordiais para se evitar o abandono e principalmente acompanhar a evolução do doente diante de um quadro de TB.

\section{DISCUSSÃO}

Como podemos observar, a maioria dos profissionais entrevistados tinham mais de 5 anos de atividade profissional e possuíam pós graduação na área de atuação. Esse dado é importante, pois o tempo de experiência profissional e a qualificação podem ser fatores que auxiliam os profissionais a desenvolverem competências e terem uma visão mais crítica sobre esse assunto.

As competências baseiam-se em: conhecimentos, habilidades e aptidões, nas quais o sujeito é capaz de mobilizar recursos cognitivos como saberes, práticas e informações em seu ambiente de trabalho, proporcionando-lhe momentos únicos e individuais de aprendizado. ' Sua aquisição depende da relação entre o conhecimento e as experiências individuais. ${ }^{10}$

Além disso, as respostas dos sujeitos da pesquisa são bastante condizentes com a literatura, pressupondo que os profissionais observam a importância do TDO,

\section{As competências}

baseiam-se em:

conhecimentos,

habilidades e

aptidões, nas quais

o sujeito é capaz de

mobilizar recursos

cognitivos como

saberes, práticas

e informações

em seu ambiente

de trabalho,

proporcionando-lhe

momentos únicos

e individuais de

aprendizado. ressaltando os benefícios tanto para o paciente como para a própria equipe, pois, enfatiza a formação do vínculo do usuário com a ESF e a consequente prevenção do abandono ao tratamento. Essa estratégia é importante para o processo de corresponsabilização e criação do vínculo. ${ }^{11}$ Ainda contribui para a adesão dos doentes e a continuidade do tratamento, uma vez que favorece o desenvolvimento de uma relação de confiança entre os sujeitos envolvidos no processo terapêutico. ${ }^{12}$

$\mathrm{O}$ vínculo entre a ESF e o doente de $\mathrm{TB}$, especialmente usuários que abandonaram o tratamento, contribui para a manutenção do cuidado por meio da monitorização das condições de saúde do doente e de sua família, considerando a saúde em seu conceito ampliado e a singularidade da pessoa que abandona o tratamento. ${ }^{13}$

Pode-se observar que apenas um dos enfermeiros associou a estratégia do TDO com a prevenção do surgimento de cepas resistentes. A interrupção do tratamento da tuberculose surge frequentemente, após o paciente apresentar melhora clíni$\mathrm{ca}$, levando assim ao surgimento de formas resistentes da tuberculose. Mostrando a associação do abandono com a multirresistência, e a necessidade do conhecimento dos profissionais de saúde em especial os enfermeiros. ${ }^{14}$

Os profissionais enfermeiros consideraram a estratégia do TDO de grande relevância, citaram sua importância quanto ao acompanhamento, identificação dos efeitos adversos, melhoria da assistência prestada, interrupção da cadeia de transmissão, acompanhamento do quadro clinico, prevenção no abandono e prevenção da resistência medicamentosa.

A maior vantagem do TDO sobre o abandono é que detecta o a situação ainda no início, possibilitando, assim, uma ação corretiva imediata. ${ }^{15}$ Quanto ao tratamento não supervisionado, o abandono só é detectado somente quando o paciente falta à consulta, ou admite não estar tomando as medicações prescritas ou quando o mesmo falha em retirar as suas medicações, o que resulta na demora na intervenção da ação da equipe de saúde. ${ }^{16}$ 


\section{artigo}

Os efeitos causados pelas drogas e as reações ao tratamento envolvem questões importantes para a adesão. Um dos fatores associados ao abandono do tratamento são os efeitos colaterais dos medicamentos, como náuseas e vômitos. ${ }^{17}$ Além disso, a adesão ao tratamento compreende também a um processo que envolve aspectos comportamentais, psíquicos e sociais, e requer decisões e responsabilidades compartilhadas de forma multidimensional, entre usuário, equipe de saúde e rede social de apoio, com uma abordagem que atenda às singularidades dos indivíduos. ${ }^{18}$

\section{CONCLUSÃO}

A realização deste estudo proporcionou um maior conhecimento a respeito da importância da realização do TDO pela perspectiva dos entrevistados. Uma vez que, evidenciou que alguns profissionais possuem conhecimento sobre importância do TDO, pois através do vínculo da equipe com o doente de tuberculose é possível saber, por exemplo, se o paciente está tomando a medicação, realizar o monitoramento das tomadas, identificar os fatores adversos, evitar o abandono e principalmente acompanhar a evolução.

No entanto, vale ressaltar conforme o observado que embora a tuberculose seja uma doença antiga, ainda existe muitas falhas na efetivação do seu controle e principalmente na batalha contra o abandono do tratamento, evidenciando a necessidade de organização do serviço na ESF, capacitação dos profissionais das unidades e também a extinção do estigma e preconceitos por parte dos usuários, familiares e população em geral.

\section{REFERÊNCIAS}

1. World Health Organization. Data Global tuberculosis report 2016. Geneva: WHO; 2016.

2. Dalcolmo M P, Andrade M K N, Picon P D. Tuberculose multirresistente no Brasil: histórico e medidas de controle. Rev. Saúde Pública [Internet]. 2007 Sep [citado em 2021 Jan 13]; 41(Suppl 1):34-42. Available from: http://www.scielo.br/scielo.php?script=sci_arttext\&pid=S0034-89102007000800006\&lng=en

3. Ministério da Saúde (BR). Secretaria de Vigilância em Saúde. Brasil livre da tuberculose: evolução dos cenários epidemiológicos e operacionais da doença. Bol Epidemiol [Internet]. 2019 mar [citado em 2021 Jan 13]; 50(9):1-18. Disponível em: https://portalarquivos2. saude.gov.br/images/pdf/2019/marco/22/2019-009.pdf

4. Ministério da Saúde (BR). Secretaria de Vigilância em Saúde Manual de Recomendações para o Controle da Tuberculose no Brasil. Programa Nacional de Controle da Tuberculose. Brasília: Ministério da Saúde; 2011.

5. World Health Organization. The Stop TB Strategy: Building on and enhancing DOTS to meet the TBrelated Millennium Development Goals. Geneva: World Health Organization; 2006.

6. World Health Organization - WHO. Stop TB Partnership. The global plan to stop TB 2006-2015 [Internet]. Geneva: World Health Organization; 2006 [cited 2021 Jan 12]. Available from: https:/ www.who.int/tb/features_archive/global_plan_to_stop_tb/en/

7. Ministério da Saúde (BR). Secretaria de Vigilância em Saúde. Departamento de Vigilância Epidemiológica.Tratamento diretamente observado (TDO) da tuberculose na atenção básica: protocolo de enfermagem. Brasília: Ministério da Saúde; 2011.

8. Bardin L. Análise de Conteúdo. Lisboa, Portugal: Edições 70, L D A; 2016.

9. Zabala, A. Como aprender e ensinar competências [recurso eletrônico]. Porto Alegre: Penso, 2014. E-PUB.

10. Fleury MTL, Fleury A. Construindo o conceito de competência. RAC. 2001;(esp):183-96.

11. Yamamura M, Martinez T R, Popolin M P, Rodrigues LBB, Freitas IM, Arcêncio RA. Famílias e o tratamento diretamente obser- vado da tuberculose: sentidos e perspectivas para produção do cuidado. Rev. Gaúcha Enferm. [Internet]. 2014 June [citado em 2021 Jan 14];35(2):60-66. Disponivel em: http://www.scielo.br/scielo.php?script=sci_arttext\&pid=S1983-14472014000200060\&lng=en. https://doi.org/10.1590/1983-1447.2014.02.42741

12. Sá, LD, Oliveira AAV, Gomes ALC, Nogueira J A, Villa TC S, Collet $N$. Cuidado ao doente de tuberculose na Estratégia Saúde da Família: percepções de enfermeiras. Rev. esc. enferm. USP [Internet]. 2012 Apr [citado em 2021 Jan 14]; 46(2): 356-363. Disponivel em: http://www.scielo.br/scielo.php?script=sci_arttext\&pid=S0080-62342012000200013\&lng=en. https://doi. org/10.1590/S0080-62342012000200013.

13. Souza KMJ, Sá LD, Palha PF, Nogueira J A, Villa TCS, Figueiredo DA. Abandono do tratamento de tuberculose e relações de vínculo com a equipe de saúde da família. Rev. esc. enferm. USP [Internet]. 2010 Dec [citado em 2021 Jan 14]; 44(4): 904-910. Disponivel em: http://www.scielo.br/scielo.php?script=sci_arttext\&pid=S0080-62342010000400007\&lng=en. https://doi. org/10.1590/S0080-62342010000400007.

14. Batista IAM, Rodrigues L, Almeida RT, Menegócio AM. O Papel do Enfermeiro na Prevenção da Farmacorresistência das Micobactérias Tuberculosas Entre os Pacientes Alcoolistas. Ensaios Cienc., Cienc. Biol. Agrar. Saúde. 2015; 19(2): 65-74, 2015.

15. Cecilio HPM, Marcon SS. Tratamento diretamente observado da tuberculose. Rev enferm UERJ, Rio de Janeiro, 2016; 24(1):e8425.

16. Ferreira AAA, Queiroz KCS, Torres KP, Ferreira MAF, Accioly $H$, Alves MSCF. Os fatores associados à tuberculose pulmonar e a baciloscopia: uma contribuição ao diagnóstico nos serviços de saúde pública. Rev Bras Epidemiol. 2005 Jun; 8(2):142-9.

17. Sá AM, Santiago LA, Santos NV, Monteiro NP, Pinto PH, Lima AM, Iwasaka-Neder PL. Causas de abandono do tratamento entre portadores de tuberculose. Rev Soc Bras Clin Med. 2017 julset;15(3):155-60.

18. Ferreira RCZ, Ramdohr Sobrinho EC, Zóia EN, Figueiredo RM. Perfil epidemiológico da tuberculose em município do interior paulista (2001- 2010). CuidArte Enferm. 2013 Jan/Jun;7(1):7-12. 